\title{
ON THE ROAD AgAIN: METAL FOAMS FIND FAVOR
}

\section{Lightweight yet stiff, metal foams are experiencing a resurgence of interest for applications ranging from automobiles to dental implants.}

\author{
John Banhart and Denis Weaire
}

$\mathrm{T}$ The three states of matter are well represented by earth, sea, and sky. God saw that it was good, and so did many generations of physics lecturers. But purity is obscurity, as Ogden Nash reminded us. The rich variety of combinations of gas, liquid, and solid that are found in nature's creations and mankind's artifacts presents us with an infinity, rather than a trinity, of possibilities (see figure 1).

Today, solid-state physics has extended its horizons to encompass all of condensed matter and the wide world of materials science. Physicists share with physical chemists and industrial engineers an eclectic interest in the exceptional properties that can emerge from mixed phases. For example, ice cream-consisting of crystalline solids, liquid, and gas-is much more than the sum of its parts. Offered separately, those parts might provide the same nutritional value, but the pleasure of consumption would be lost. Here, structure is as important as composition, and it owes more to culinary artifice than to the laws of thermodynamics. In the metastable world of mixed-phase structures, science meets art. The current interest in mixed phases was heralded in the 1950s by the MIT metallurgist Cyril Stanley Smith, as recounted in his testament, $A$ Search for Structure. ${ }^{1}$

Smith was particularly attracted to the elegant liquid-gas system that is called a foam, a prototype for much of materials science. A foam is typically disordered, and each sample is a product of its particular history, yet its internal local arrangements are not arbitrary. They conform to rules, dictated by surface tension, that were expounded by the blind Belgian physicist Joseph Plateau in the 19th century. Those rules require that only three of the thin films that separate bubbles can meet on a line (called a Plateau border), and only four of those lines can meet at a point. Films and lines meet symmetrically, at equal angles. The rules strictly apply only in the limit of zero liquid fraction, but most foams have low enough liquid fraction to conform well to them. ${ }^{2}$

The beautiful structure described by Plateau is common to most liquid foams, and hence also to the solid foams that result from freezing them. Solid foams encountered in everyday life include polyurethane and polystyrene

JOHN BANHART (banhart@hmi.de) is a professor of materials science at the Technical University of Berlin and head of the department of materials science at the Hahn-Meitner Institute in Berlin, Germany. DENIS WEAIRE (dweaire@tcd.ie) is the Erasmus Smith Professor in the department of physics at Trinity College Dublin in Ireland. products for cushioning, packaging, and insulation. Many other materials, such as ordinary glass, can be foamed. Even metals are produced as foams. A comparatively new entry in the foam catalog, metal foams are attracting a lot of attention as promising applications are identified. ${ }^{3}$

\section{Light, stiff, crushable}

A metal foam consists primarily of a network of thin, frozen Plateau borders meeting at junctions that usually have the prescribed tetrahedrally symmetric form. The relative density of a solid metal foam (overall density divided by that of its solid constituent) is typically less than $15 \%$.

In their classic book on solid foams, Lorna Gibson and Michael Ashby pursued many simple scaling relations for physical properties of relevance to engineering. ${ }^{4}$ An important example is the Young's modulus, which determines the stiffness of a panel or a beam. Assuming that the thin, strutlike Plateau borders make the dominant contribution to elastic properties, the Young's modulus $E$ is predicted to scale as

$$
E \propto(\text { relative density })^{1.5} .
$$

For real foams, the empirical law is closer to

$$
E \propto(\text { relative density })^{2} .
$$

The stiffness of a panel-that is, its resistance to bending-varies as its thickness cubed times the Young's modulus of the material. So if a dense metal panel is replaced by a foam panel of the same weight but that is five times thicker, its bending stiffness increases by the same factor of five. The stiffness-to-weight ratio is often an important figure of merit for the structural engineer.

Metal foams offer another benefit, having to do with their deformation under high loads. Different types of solid foam behave in different ways when large deformations are imposed and the network buckles. In a polyurethane cushion, the struts undergo the so-called Euler buckling of an elastic beam under compression (such buckling is what is observed when a meter stick is squeezed from both ends). Although deformed, the original structure is recovered on removal of the applied load, and the material remains undamaged. In contrast, metal foams fail irreversibly. Depending on the type of alloy the foam is made of, the cells suffer brittle fracture, deform plastically without breaking, or show an even more complicated deformation pattern. In each case, there is a certain yield stress at which strongly nonlinear effects enter into play. 
FIGURE 1. MiXED-PHASE STATES such as solid and liquid foams are widespread in nature, the laboratory, and the production line.

Most foams collapse gradually under the critical compressive load until a high degree of compaction is achieved. That process absorbs a great deal of mechanical energy, and therein lies the second main attraction of metal foams, which have a high yield stress compared to polymer foams. Add in the compatibility of metal foams with much of existing automobile technology of metal forming and welding, and the motivation for current research becomes obvious. The ultimate fate of some metal foam is to be crushed in a crash, to be one of the sacrificial elements of modern vehicle design that are deliberately intended to collapse in order to save passengers from experiencing something similar. The material might be termed heavy-duty polystyrene, because 1 cubic centimeter of aluminum foam can absorb up to 10 joules of mechanical energy if crushed to $20 \%$ of its original length.

\section{But can we make it?}

In 1943, Benjamin Sosnick attempted to foam aluminum with mercury. ${ }^{5}$ He first melted a mix of $\mathrm{Al}$ and $\mathrm{Hg}$ in a closed chamber under high pressure. The pressure was released, which led to vaporization of the $\mathrm{Hg}$ at the melting temperature of $\mathrm{Al}$ and to the formation of a foam.

Less hazardous processes were developed in the 1950 s with the realization that liquid metals could be more easily foamed if they were pretreated to enhance their viscosity. Such enhancement could be done by oxidizing the melt or by adding oxide particles. William Elliott and Stuart Fiedler at Bjorksten Research Laboratories (BRL) in Madison, Wisconsin, developed an aluminum foaming process for the US Navy in the late 1950s. BRL subsequently entered into an agreement with the LOR Corp to develop commercial uses for foamed aluminum. A pilot plant was constructed at BRL to produce $4^{\prime} \times 8^{\prime} \times 1^{\prime \prime}$ panels. Potential uses, such as crush bumpers for cars, were also investigated. In the late $1960 \mathrm{~s}$, the entire operation was sold to the Ethyl Corp and the pilot plant was moved to Baton Rouge, Louisiana. BRL continued for several years to investigate methods for foaming other metals such as lead and zinc.

Two methods for foaming metals were used in those early days, and they are still current today. In the first, gas is injected continuously to create foam. In the second method, gas-releasing propellants-akin to the blowing agents of the plastic foam industry or indeed the yeast of the baker (see figure 2)-are added to the melt.

Aluminum was found to be particularly amenable to foam production. Around 1972, the Ethyl Corp produced aluminum foam of remarkably high quality that was given to the Ford Motor Co for evaluation. Why was this initial development unsuccessful? Certainly it was not the time for lightweight materials in the era of seemingly unlimited energy supply, and issues of safety and recycling were not as important as they are now. Perhaps also the disordered nature of the material caused some raised eyebrows in quality control. Whatever the reason, the excitement and the level of R\&D activities both declined after 1975 .

The end of the 1980s saw a resurgence in metal foam research throughout the world. Japanese engineers at

Mists

Liquid foams GAS

LIQUID Smokes Solid foams

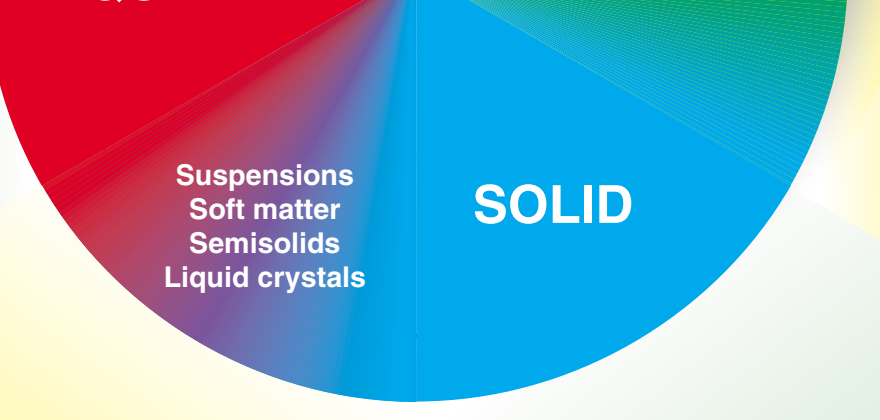

Shinko Wire Co developed what is now known as the Alporas process (see the box on page 39). Norsk Hydro in Norway and Alcan Corp in Canada independently developed a foaming process for particle-stabilized melts. In 1990, an old compacted-powder foaming process developed in the late 1950s by Benjamin Allen at the United Aircraft Corp in Delaware was rediscovered by German physicist Joachim Baumeister and brought to a considerable level of sophistication at the Fraunhofer Institute in Bremen, Germany. That process uses powder blends that are first compacted and then foamed by remelting. Those methods and other variants have been continually refined up to the present day.

The accompanying box summarizes processes that have the potential for making large slabs and structural components. ${ }^{6}$ Most of the resulting foam products have closed cells. A certain degree of open porosity can be achieved by various post-foaming treatments such as rolling. Alternatively, a quite different process, that of investment casting, produces metal foams as replicas of open-celled plastic foams. Cast open porous structures are not foams in the strict sense, but rather sponges. ${ }^{6,7}$

\section{The physics of metal foaming}

Making a foam from compacted powder mixtures involves five stages, all of which offer opportunities for research

- Making the precursor, by compacting a mix of metal powder and gas-releasing blowing-agent powder. The densification has to be such that each blowing-agent particle is encapsulated in the metallic matrix. Evolving gas must not be allowed to escape through some residual porosity.

- Initial pore formation. On heating, gas evolves in the precursor and forms the nuclei of pores. Depending on the temperature ranges over which the blowing agent decomposes and the alloy melts, nucleation may occur in the solid, semisolid, or liquid state.

- Pore inflation. Driven by the ongoing gas supply from the decomposing blowing agent, the nuclei are enlarged and come together to form the foam. Whereas temperature is usually constant in the foaming of aqueous liquids, it is not 


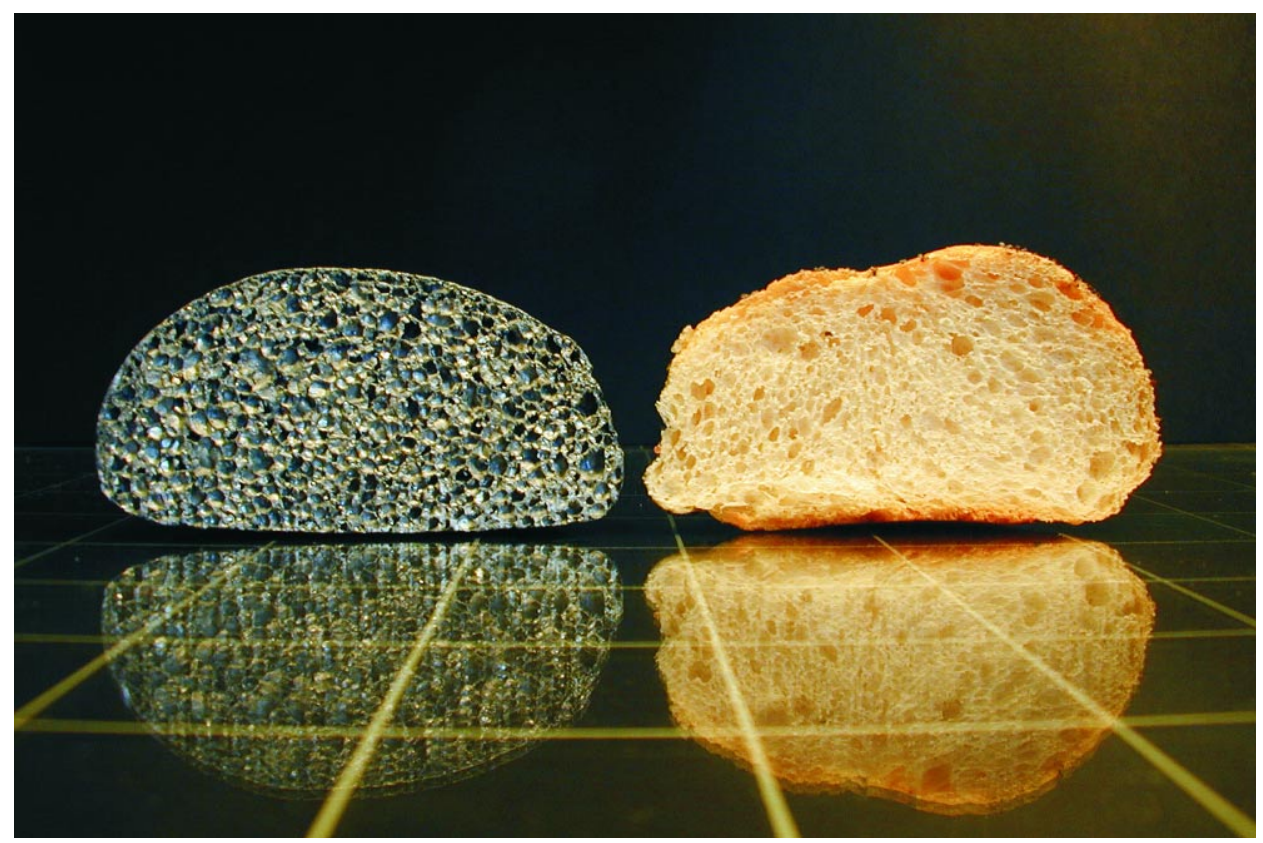

FIGURE 2. ZINC FOAM and bread roll, both $8 \mathrm{~cm}$ wide. The pores in the metal foam were formed by hydrogenreleasing chemicals; those in the bread by $\mathrm{CO}_{2}$-releasing yeast.

Solidification. To keep the unstable foam from collapsing, the metal has to be solidified quickly at exactly the right time. Sudden cooling can lead to deformations of the films or create cracks in the cell walls, especially if heat flow is nonuniform.

Little can be done to speed up the heat extraction so here because the precursor is being heated continuously. - Foam degradation. While pores are being inflated, degradation begins. The downward flow of liquid through Plateau borders under gravity causes drainage, and bubbles coalesce as films become thin and unstable.

to prevent collapse. The remaining option is to slow the drainage by increasing viscosity, which is the primary role of many additives that are used in commercial processes. Usually such additives are finely dispersed nonmetallic components such as oxides or carbides that remain solid

\section{Industrial Processes for Metal Foam Production}

$\mathrm{M}$ any different processes have been developed for producing metal foams. The five most significant ones are shown in the figure.

In the Hydro/Alcan process, liquid metals-usually aluminum alloys-are foamed directly by injecting gas. Silicon carbide is commonly added in volume fractions as high as $10-20 \%$ to stabilize the foam. Foam can be continuously drawn off the surface of the melt on a conveyor belt, and slabs with a cross section of $100 \times 400 \mathrm{~mm}^{2}$ can be produced in lengths up to $16 \mathrm{~m}$. Foam densities range from 0.07 to $0.54 \mathrm{~g} / \mathrm{cm}^{3}$, with average bubble sizes of 3 to $25 \mathrm{~mm}$. The Canadian company Cymat is industrializing the process. The Combal process, developed by the Light Metal Competence Center and Hütte Kleinreichenbach, both in Austria, is similar and produces nearly uniformly sized pores.

The Alporas process foams a pool of aluminum by adding titanium hydride powder as a blowing agent. The aluminum melt is thickened by adding calcium, which forms fine mixed oxides in the melt. The foaming vessel in use at Shinko Wire Co in Japan produces blocks of size $2050 \times 650 \times 450 \mathrm{~mm}^{3}$.

The Gasar/Lotus process foams metal by supersaturating a liquid with hydrogen under high pressure and temperature, after which the liquid is solidified and gas precipitates into largely elongated pores. "Gasar" is a Russian acronym that stands for "gas-reinforced." The process was developed by a Ukrainian scientist more than 10 years ago, and has recently been picked up by researchers at the University of Osaka, who call their material "lotus-type foam" because the morphology of the material resembles that of a lotus root.

The Alulight/Foaminal process starts from a compacted mixture of metal and blowing-agent powders, such as aluminum and titanium hydride. Remelting the precursor leads to in situ gas evolution within the metal as the blowing agents release hydrogen gas. Alulight and Foaminal are commercial trade names for the resulting solid material. With this process, closed molds can be filled with foam, and structural foam parts of complex shape can be manufactured. Shaped sandwich panels with two dense face sheets and a cellular core can also be made. ${ }^{6,13}$

The Formgrip process starts from a melt in which blowingagent powder is dispersed. The melt is then solidified to form a solid precursor that can later be foamed by remelting. ${ }^{14}$ The name "Formgrip" stands for "foaming of reinforced metals by gas release in precursors."

\section{Metal foams}

Foaming route

Stabilization

Gas source

Process name

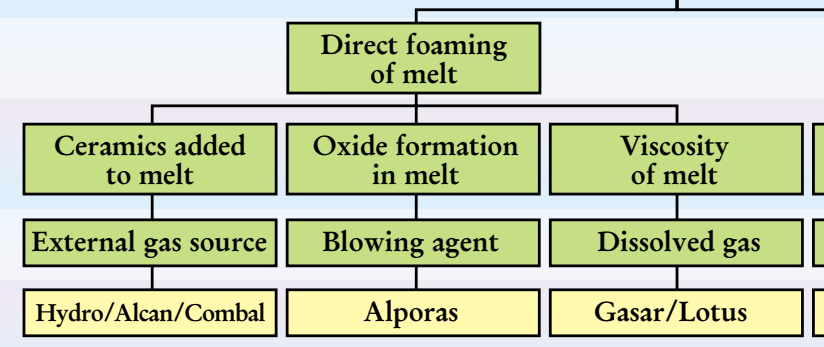

\begin{tabular}{|c|c|}
\hline $\begin{array}{c}\text { Indirect foaming } \\
\text { via precursor }\end{array}$ \\
\begin{tabular}{|c|} 
Oxides in \\
compacted powder
\end{tabular} \\
\hline Blowing agent & Ceramics added \\
to melt
\end{tabular}



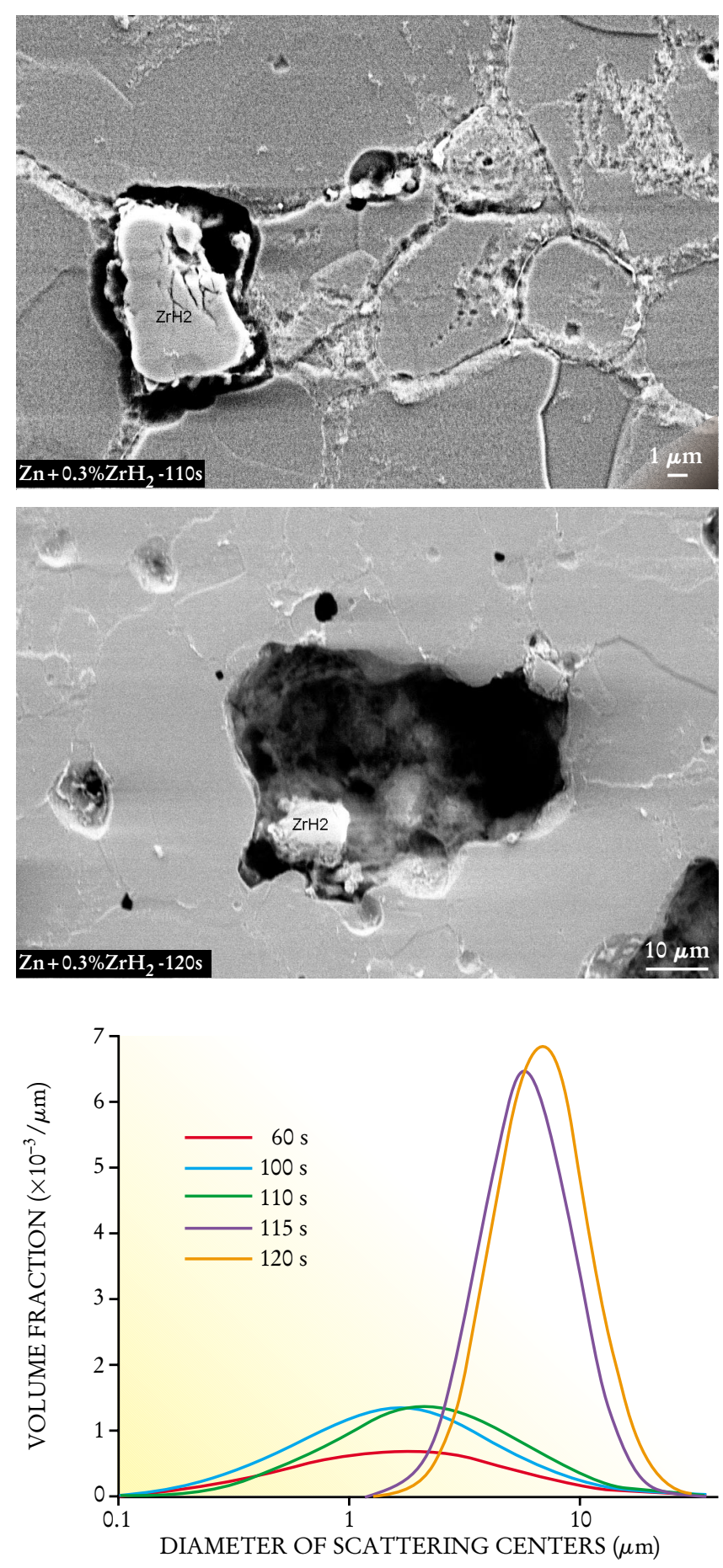

in the liquid and make it highly viscous. Another trick is to use alloy compositions for which the melt has a "mushy" semisolid character in a certain temperature range. Popular choices are aluminum-silicon or aluminum-magnesium alloys. Additives are often thought to play a further role as surfactants, together with dissolved oxygen, but such behavior is not well established. The notional surfactants are presumed to stabilize the thin films.

A range of sophisticated physical instrumentation has already been brought to bear on the compacted powder foaming process. Pore nucleation can be investigated by quenching the foaming sample before foam formation sets in. Ultrasmall-angle neutron scattering (USANS) can measure the pore size distribution as an average over the
FIGURE 3. FOAM PORES undergo sudden growth spurts. The top scanning electron microscopic image shows a zinc foam after 110 seconds of foaming at $440^{\circ} \mathrm{C}$. The bottom image, taken only 10 seconds later, shows a greatly increased pore size. The graph shows the pore size distributions measured by ultrasmall-angle neutron scattering (USANS) at various times after the foaming started. ${ }^{8}$

entire sample. ${ }^{8}$ And scanning electron microscopy can probe individual pores within the sample.

The scanning electron microscope images of figure 3 reveal the mechanism of initial pore formation in zinc foamed with zirconium hydride as a blowing agent. After 110 seconds of heating, $\mathrm{ZrH}_{2}$ particles are surrounded by an annular void formed by the evolving hydrogen gas. Just ten seconds later, the voids have grown considerably. Most blowing-agent particles are found on the walls of bubbles. Bubbles can also be formed in the absence of a particle, due to the diffusion of hydrogen gas through the metal. The pore size distributions measured by USANS confirm this picture of bubble growth: The distribution function is centered around $2 \mu \mathrm{m}$ for short foaming times and gradually moves to higher values before rapid pore growth sets in.

Zinc shows a comparatively simple behavior because the decomposition temperature of the blowing agent and the melting point of metal are nearly the same (about $420^{\circ} \mathrm{C}$ ). Therefore, pore formation occurs in the liquid state, and the bubbles are spherical. In aluminum foamed with titanium hydride, the situation is different: Gas evolution starts in the solid state, and so the initial porosity has a more complex morphology.

The rapidly developing technique of synchrotron x-ray radioscopy enables direct in situ monitoring of the evolution of bubbles. In an experiment at the European Synchrotron Radiation Facility in Grenoble, France, ${ }^{9}$ foams were generated in a furnace equipped with water-cooled $\mathrm{Al}$ windows through which the synchrotron x-ray beam could pass. Absorption radiographs with spatial resolutions between 10 and $40 \mu \mathrm{m}$ were captured at frequencies between 2 and $18 \mathrm{~Hz}$ with an electronic detector system. The entire foaming experiment took a few minutes, corresponding to 500-900 radiographs.

If the chosen samples have a thickness of only a few bubble diameters in the beam direction, a wealth of details can be detected with x rays. Figure 4, for instance, shows the rupture of a cell wall. These beautiful pictures rival anything that has been produced in the more traditional context of aqueous foams.

The solidification process can also be monitored by cooling the foam while observing it with x-ray radioscopy. Moreover, one can use molds made of thin titanium foil, which absorbs $\mathrm{x}$ rays only weakly, to visualize foam flow.

Because drainage can make metal foam formation a race against time, the microgravity environment of space offers a promising new dimension for experiments. Some experiments have been carried out already on parabolic flights, but the long-term goal of a research effort of ours, funded by the European Space Agency, is to foam metals on board the International Space Station.

\section{The future of metal foams}

The second surge of research on these new materials has begun to produce much more reliable and homogeneous foams.

In the latest development, Dietmar Leitlmeier and 


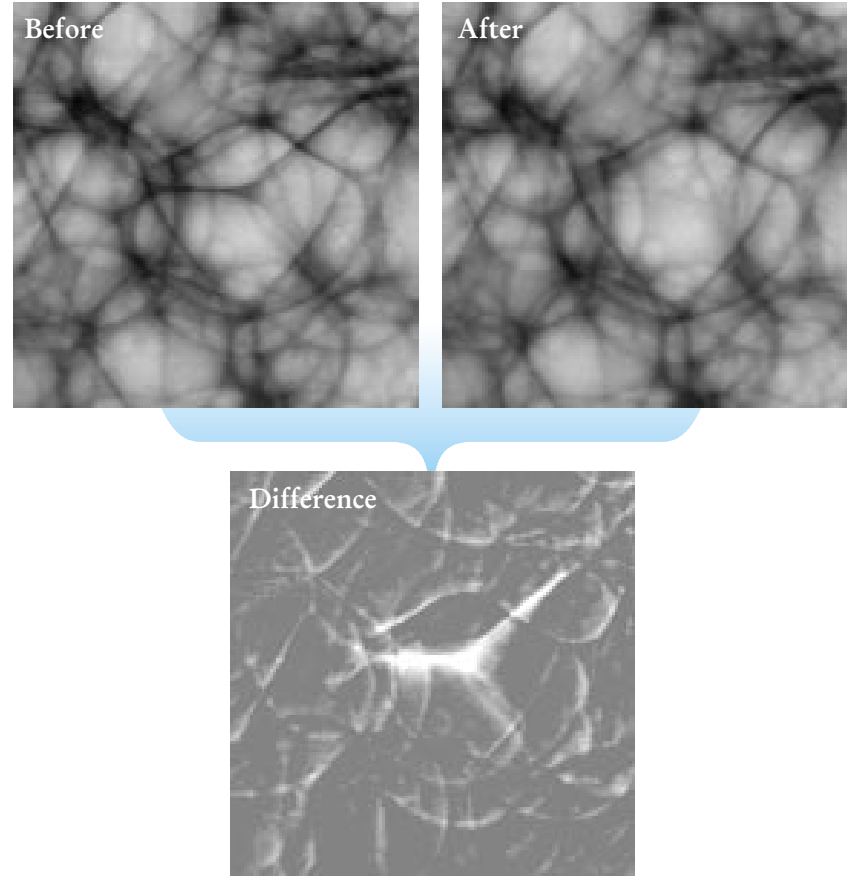

Figure 4. Cell RUPTURE observed in a foam of AlSi7 (aluminum with $7 \%$ silicon by weight). Two successive radiographs (top), $500 \mathrm{~ms}$ apart, show an area of $4.5 \times 4.5 \mathrm{~mm}^{2}$. The foam structure suddenly changes within that time span. The change-the rupture of three cell membranes-is highlighted by calculating the difference between the two pictures (bottom). ${ }^{9}$

colleagues at the Light Metal Competence Center (LKR) and Hütte Kleinreichenbach (HKB), both located in Austria, have found a new way to produce extremely uniform aluminum foam structures stabilized with ceramic particles. The trick is a novel bubble generation device that allows the creation of nearly uniform-sized bubbles. The trade name Combal has been given to the foamed material. Figure 5 shows a tomographic image of a Combal sample and the corresponding size distribution function.

Metal foams are finding their way into many new applications. A recent design guide provides a comprehensive framework for evaluating applications. ${ }^{10}$ Proceedings of the latest conferences ${ }^{11,12}$ and a new handbook on metallic foams ${ }^{7}$ illustrate the wide range of possibilities, which arise in the automotive, aerospace, nautical, railway, building, civil engineering and medical industries. For example, shipbuilders could use large panels of aluminum foam for doors, escape hatches, or walls, exploiting the high stiffness-to-weight ratio, vibration damping capacity, and nonflammability of the material. In industrial settings, foam-filled columns or sandwich panels could replace conventional dense metal, such as in rotating printing rolls or quickly moving platforms or crossbeams in machines, in order to reduce their inertia and to damp vibrations. The biomedical industry could use foams based on titanium as dental implants, since titanium is biocompatible and the elastic properties of the foam can be adapted to the modulus of the bones through selection of appropriate porosities. ${ }^{6}$

The automotive industry has been most active in metal foam R\&D in recent years. The German automobile
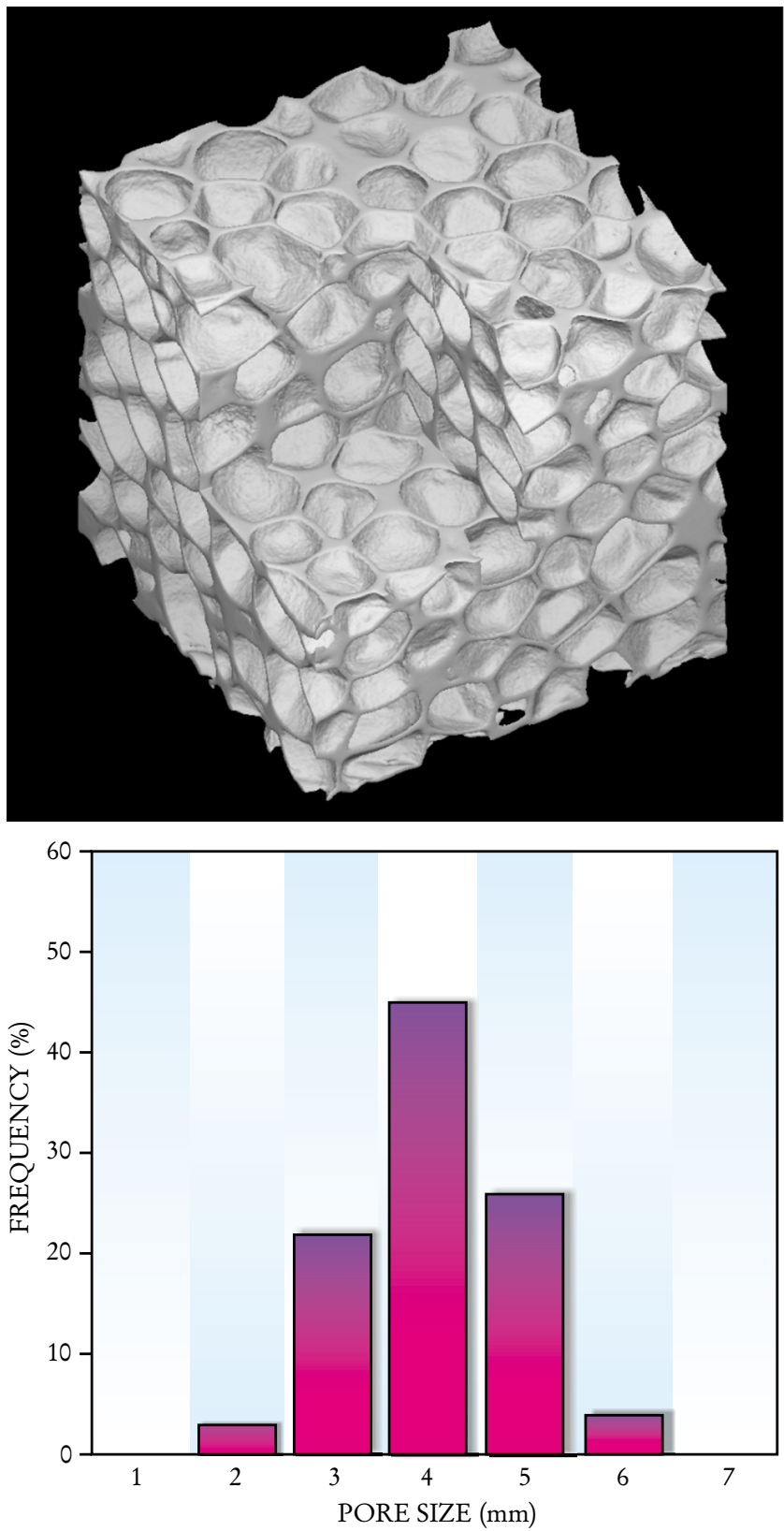

FIgURE 5. STATE-OF-THE-ART FOAM. This foam of the common alloy aluminum 6061, stabilized with alumina particles, has pores with a very narrow distribution of sizes, as shown in the bar graph. The tomographic image is $34 \mathrm{~mm}$ on a side. (Courtesy of Light Metal Competence Center and Hütte Kleinreichenbach).

supply company Wilhelm Karmann has developed a foam sandwich technology in collaboration with the Fraunhofer Institute in Bremen. ${ }^{7}$ One of their aluminum foam sandwich (AFS) components is shown in figure 6: a flat sandwich panel with a foamed aluminum core between two aluminum face sheets. More complex shapes can be manufactured as well without using any adhesives. Such components are damage tolerant and easy to integrate into a car's aluminum structural frame. Incorporated into prototypes that are currently under testing, metal foams are truly back on the road.

Work on the AFS technology started about eight years 


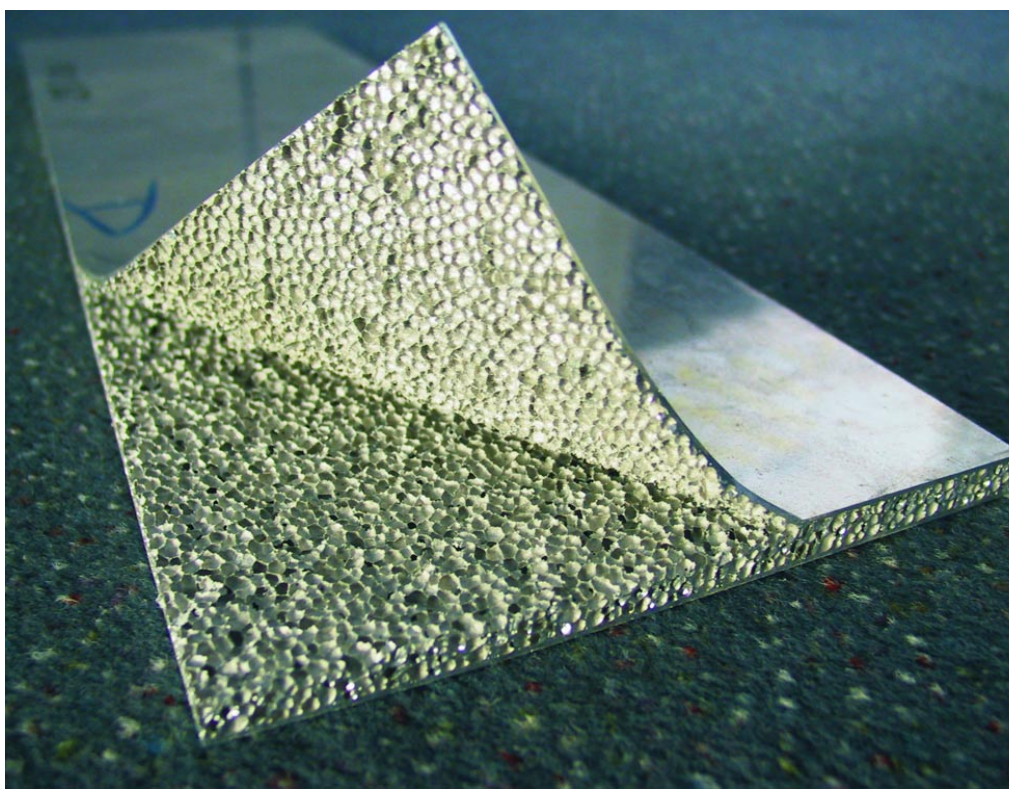

FIGURE 6. LIGHTWEIGHT ALUMINUM FOAM SANDWICH.

The face sheet has been partially torn off to show the quality of bonding between foam and sheet (Courtesy of Wilhelm Karmann GmbH.)

ago. A rule of thumb says that it usually takes 15 years for a new technology to develop from a laboratory curiosity to a marketed product; the next few years may see the completion of that evolution for AFS. Without such a major implementation, industry and the scientific community may again lose interest in metal foams. But for the time being, the road ahead looks clear.

We would like to thank Dietmar Leitlmeier (LKR), Mike Maloney (BRL) and Wolfgang Seeliger (Karmann) for providing information and pictures.

\section{References}

1. C. S. Smith, A Search for Structure: Selected Essays on Science, Art, and History, MIT Press, Cambridge, Mass. (1981).

2. D. Weaire, S. Hutzler, The Physics of Foams, Clarendon Press, New York (1999).

3. For the latest developments on metal foams, see http://www.metalfoam.net.

4. L. J. Gibson, M. F. Ashby, Cellular Solids: Structure and Properties, Cambridge U. Press, Cambridge, UK (1997).

5. B. Sosnick, "Process for Making Foamlike Mass of Metal," US Patent 2,434,775 (20 January 1948).

6. J. Banhart, Prog. Mater. Sci. 46, 559 (2001).

7. H. P. Degischer, B. Kriszt, eds., Handbook of Cellular Metals: Production, Processing, Applications, Wiley, New York (2002).

8. J. Banhart, D. Bellmann, H. Clemens, Acta Mater. 49, 3409 (2001).

9. J. Banhart, H. Stanzick, L. Helfen, T. Baumbach, Appl. Phys. Lett. 78, 1152 (2001).

10. M. F. Ashby, A. G. Evans, N. A. Fleck, L. J. Gibson, J. W. Hutchinson, H. N. G. Wadley, Metal Foams: A Design Guide Butterworth-Heinemann, Boston (2000).

11. J. Banhart, M. F. Ashby, N. A. Fleck, eds., Metal Foams and Porous Metal Structures (Proc. MetFoam '99), MIT-Verlag, Bremen, Germany (1999).

12. J. Banhart, M. F. Ashby, N. A. Fleck, eds., Cellular Metals and Metal Foaming Technology (Proc. MetFoam 2001), MIT-Verlag, Bremen, Germany (2001).

13. F. Baumgärtner, I. Duarte, J. Banhart, Adv. Eng. Mater. 2, 168 (2000).

14. V. Gergely, T. W. Clyne, Adv. Eng. Mater. 2, 175 (2000). 